\title{
Transformations and Lorenz Curves: Sufficient and Necessary Conditions
}

\author{
Johan Fellman \\ Hanken School of Economics, Helsinki, Finland \\ Email: fellman@hanken.fi
}

Received 10 May 2016; accepted 3 June 2016; published 6 June 2016

Copyright (C) 2016 by author and Scientific Research Publishing Inc.

This work is licensed under the Creative Commons Attribution International License (CC BY).

http://creativecommons.org/licenses/by/4.0/

(c) (i) Open Access

\begin{abstract}
In this study, we reconsider the effect of variable transformations on income inequality. Under the assumption that the theorems should hold for all income distributions, earlier given sufficient conditions are also necessary. Different versions of the conditions are compared. Furthermore, one can prove that the assumption of continuity of the transformations can be implicitly included in the necessary and sufficient conditions, and hence, it can be dropped from the assumptions. The effects of two transformations on income inequality are compared.
\end{abstract}

\section{Keywords}

Discontinuity, Income Distribution, Income Inequality, Lorenz Dominance, Tax Policy, Transfer Policy

\section{Introduction}

It is a well-known fact that variable transformations are valuable in considering the effect of tax and transfer policies on income inequality. The transformation is usually assumed to be positive, monotone increasing and continuous. Under the assumption that the theorems should hold for all income distributions, conditions given earlier are both necessary and sufficient [1] [2]. Hemming and Keen [3] have given an alternative version of the conditions. Recently, Fellman [2] [4] also discussed discontinuous transformations. One general result is that continuity is a necessary condition if the transformation should preserve or reduce income inequality. If the transformation is considered as a tax or a transfer policy, the transformed variable is either the post-tax or the posttransfer income. In this study, we reconsider the effect of variable transformations on the redistribution of income. Two transformations are studied and their effects on income inequality are compared.

\section{Properties of a Transformed Variable}

Consider the income $X$ with the cumulative distribution function $F_{X}(x)$, the frequency distribution $f_{X}(x)$, the 
mean $\mu_{X}$, and the Lorenz curve $L_{X}(p)$. We assume that $X$ is defined for $x \geq 0$ and that $f_{X}(x)$ is continuous. Furthermore, we consider the transformation $Y=g(X)$, where $g(\cdot)$ is non-negative and monotone increasing. A fundamental theorem concerning the effect of income transformations on Lorenz curves was first given by Fellman [5], Jakobsson [1], and Kakwani [6] and later by Fellman [7] [8]. Hemming and Keen [3] gave a new condition for the Lorenz dominance. We have

Theorem 1. Let $X \geq 0$ be a random variable with an arbitrary continuous frequency distribution $f_{X}(x)$, mean $\mu_{X}$, and the Lorenz curve $L_{X}(p)$. Let $g(x)$ be positive, continuous, and monotone increasing, let $Y=g(X)$, and let $\mu_{Y}=E(g(X))$ exist. Then, the Lorenz curve $L_{Y}(p)$ of $Y$ exists and the following results hold:

1) $L_{Y}(p) \geq L_{X}(p)$ if $g(x) / x$ is monotone decreasing

2) $L_{Y}(p)=L_{X}(p)$ if $g(x) / x$ is constant

3) $L_{Y}(p) \leq L_{X}(p)$ if $g(x) / x$ is monotone increasing.

Proof: From the fact that

$$
L_{Y}(p)=\frac{1}{\mu_{Y}} \int_{0}^{x_{p}} g(x) f(x) \mathrm{d} x \leq \frac{1}{\mu_{Y}} \int_{0}^{\infty} g(x) f(x) \mathrm{d} x=1,
$$

it follows that $L_{Y}(p)$ exists.

The case 2) follows immediately from the fact that the Lorenz curve remains when linear transformation is performed. Consider the difference

$$
\begin{aligned}
D(p) & =L_{Y}(p)-L_{X}(p)=\frac{1}{\mu_{Y}} \int_{0}^{x_{p}} g(x) f(x) \mathrm{d} x-\frac{1}{\mu_{X}} \int_{0}^{x_{p}} x f(x) \mathrm{d} x \\
& =\int_{0}^{x_{p}}\left(\frac{g(x)}{\mu_{Y}}-\frac{x}{\mu_{X}}\right) f(x) \mathrm{d} x=\int_{0}^{x_{p}} \frac{x}{\mu_{Y}}\left(\frac{g(x)}{x}-\frac{\mu_{Y}}{\mu_{X}}\right) f(x) \mathrm{d} x .
\end{aligned}
$$

By definition, $D(0)=D(1)=0$. First, we assume that $\frac{g(x)}{x}$ is continuous and monotone decreasing for $x>0$. Then $\left(\frac{g(x)}{x}-\frac{\mu_{Y}}{\mu_{X}}\right)$ attains zero only once, being first positive and then negative. Hence, the difference $D(p) \geq 0$ and the case 1) is proved.

For the case 3), $\frac{g(x)}{x}$ is monotone increasing for $x>0$. Also in this case $D(0)=D(1)=0$. The difference $\left(\frac{g(x)}{x}-\frac{\mu_{Y}}{\mu_{X}}\right)$ attains zero only once, being first negative and then positive. Hence, $D(p) \leq 0$ and the case 3 ) is proved.

If we consider tax policies, $x$ is the pre-tax income and the function $g(x)$ is the after-tax income and the ratio $1-g(x) / x$ is the relative tax. If the ratio $g(x) / x$ is monotonically decreasing, $1-g(x) / x$ is monotone increasing and the tax policy is progressive. Hence, Theorem 1 1) states the well-known result that progressive taxes reduce income inequality.

In addition, if we consider income increases and that $g(x)$ is the increased income and that 1) holds then the income increase reduces the income inequality.

According to Theorem 1, we obtain in 1) a sufficient condition that the transformation $g(x)$ results in a new income distribution, which Lorenz dominates the initial one. What can be said about necessary conditions? If we analyze the proof of Theorem 1, we observe that the difference

$$
D(p)=L_{Y}(p)-L_{X}(p)=\int_{0}^{x_{p}} \frac{x}{\mu_{Y}}\left(\frac{g(x)}{x}-\frac{\mu_{Y}}{\mu_{X}}\right) f(x) \mathrm{d} x
$$

plays a central role. For a transformation $g(x)$ for which the quotient $g(x) / x$ is not monotone decreasing 
for all $x>0$, an income distribution $f_{X}(x)$ can be chosen so that the result in the proof holds, i.e. dominance is obtained. We have only to choose $f_{X}(x)$ and $g(x)$ so that $D(p)$ is non-negative for all $p$. For example if the quotient $g(x) / x$ is both increasing and decreasing we choose the distribution $f_{X}(x)$ so that $f_{X}(x)$ is positive only in an interval where $g(x) / x$ is monotone decreasing.

The sufficient condition of Hemming and Keen [3] is (with our notations) that for a given distribution $f_{X}(x)$ the function $g(x)$ crosses the line $\frac{\mu_{Y}}{\mu_{X}} x$ once from above. The Hemming-Keen condition is equivalent with the condition that $g(x) / x$ crosses the level $\frac{\mu_{Y}}{\mu_{X}}$ from above, which is easier to compare with ours. We observe that if their condition holds then the integrand in (2) starts from positive values, changes its sign once, and ends up with negative values.

If we demand necessary conditions, they must be formulated as a condition that holds for all income distributions $f_{X}(x)$. The condition of Hemming and Keen must be that $g(x)$ must satisfy the condition "crossing once from above for all distributions $f_{X}(x)$ " [3]. We start with the condition in Theorem 11$)$ and prove that it is also necessary. This can be proved in the following way ([1] [9], p. 189). Let a transformation $g(x)$ satisfy the initial conditions (positive, continuous, and monotone increasing) and let $g(x) / x$ be increasing within some interval $(0<a<x<b<\infty)$. Now, we prove that there exists an income distribution $f_{X}(x)$ such that the transformed variable $Y=g(X)$ does not Lorenz dominate the initial variable $X$.

Consider an income distribution

$$
f(x)=\left\{\begin{array}{ccc}
0 & 0<x<a \\
f_{0}(x) & \geq 0 & a \leq x \leq b \\
0 & b<x
\end{array}\right.
$$

For the pair $(f(x), g(x))$, Theorem 13 ) holds and the transformation results in a new variable $Y$, which is Lorenz dominated by the initial variable $X$. This result indicates that if $g(x) / x$ is monotone increasing even in a short interval, then there are income distributions such that the transformation $g(x)$ cannot result in Lorenz dominance. Hence, if we demand that, for all distributions $f(x)$, the transformed variable $Y=g(X)$ shall Lorenz dominate $X$ then the condition in Theorem 11$)$ is necessary. In the example considered above, the Hemming-Keen condition is not satisfied. Consequently, if $g(x) / x$ is not monotone decreasing then there are distributions for which the Hemming-Keen condition does not hold. On the other hand, if we assume that $g(x) / x$ is monotone decreasing then $g(x)$ satisfies the condition "crossing once from above for every distribution $f(x)$ ". Hence, our condition and the Hemming-Keen condition are equivalent as necessary conditions. In a similar way, we can prove that if the other results in Theorem 1 should hold for every income distribution the conditions in 2) and in 3) are also necessary.

Now, we follow [8] and drop the assumption that $g(x)$ is continuous and consider discontinuous functions. What can be said about the case that $g(x)$ is discontinuous? Assume that $g(x)$ is still positive and monotone increasing and satisfies the condition that $E(g(x))$ exists for every stochastic variable $X$, whose distribution $f(x)$ satisfies the general conditions given above, then the discontinuities can only consist of denumerable finite positive jumps. Now we will prove that if there exists one such jump there exists at least one distribution $f(x)$ such that the transformation $Y=g(X)$ does not Lorenz dominate the initial variable $X$.

Let $a$ be a discontinuity point such that $\lim _{x \rightarrow a-} g(x)=g_{0}$ and $\lim _{x \rightarrow a+} g(x)=g_{0}+d$ where $d>0$. If $g(x)$ should be monotone increasing, we have to assume that $g_{0} \leq g(a) \leq g_{0}+d$. Let $y \leq a$ and $z \geq a$, then

$$
\lim _{y \rightarrow a-} \frac{g(y)}{y}=\frac{g_{0}}{a}<\frac{g_{0}+d}{a}=\lim _{z \rightarrow a-} \frac{g(z)}{z}
$$

Hence, we note that the quotient $g(x) / x$ cannot be monotone decreasing within a short interval $\left(a-\frac{1}{2} h, a+\frac{1}{2} h\right)$. Choose $h>0$ so small that the point $a$ is the only discontinuity point within the interval 
$\left(a-\frac{1}{2} h, a+\frac{1}{2} h\right)$ (later we may reduce $h$ even more).

Consider the uniform distribution

$$
f_{X}(x)= \begin{cases}0 & x<a-\frac{1}{2} h \\ \frac{1}{h} & a-\frac{1}{2} h \leq x \leq a+\frac{1}{2} h \\ 0 & x>a+\frac{1}{2} h\end{cases}
$$

For this variable $X$, the mean is $\mu_{X}=E(X)=a$. For the transformed variable $Y=g(X)$, the mean is

$$
\mu_{Y}=E(Y)=\frac{1}{h} \int_{a-\frac{1}{2} h}^{a} g(x) \mathrm{d} x+\frac{1}{h} \int_{a}^{a+\frac{1}{2} h} g(x) \mathrm{d} x=\frac{1}{2}\left(g\left(\alpha_{1}\right)+g\left(\alpha_{2}\right)\right)
$$

where $\left(a-\frac{1}{2} h<\alpha_{1}<a\right)$ and $\left(a<\alpha_{2}<a+\frac{1}{2} h\right)$.

If $h \rightarrow 0$ then $\alpha_{1} \rightarrow a-, g\left(\alpha_{1}\right) \rightarrow g_{0}, \alpha_{2} \rightarrow a+, g\left(\alpha_{2}\right) \rightarrow g_{0}+d$, and consequently, $\mu_{Y} \rightarrow g_{0}+\frac{1}{2} d$.

Assume that we choose $h$ so small that $g_{0}+\frac{1}{4} d<\mu_{Y}<g_{0}+\frac{3}{4} d$. Consider now

$$
D(p)=L_{Y}(p)-L_{X}(p)=\int_{a-\frac{1}{2} h}^{a+\frac{1}{2} h} \frac{x}{\mu_{Y}}\left(\frac{g(x)}{x}-\frac{\mu_{Y}}{\mu_{X}}\right) \frac{1}{h} \mathrm{~d} x
$$

To obtain Lorenz dominance, the integrand must start from positive (non-negative) values and then change its sign once and become negative in such a manner that the difference $D(p)$ starts from zero and then attains positive values, whereupon it decreases back to zero.

The sign of the integrand depends on the factor $\left(\frac{g(x)}{x}-\frac{\mu_{Y}}{\mu_{X}}\right)$, which starts from the value

$$
\frac{g\left(a-\frac{1}{2} h\right)}{a-\frac{1}{2} h}-\frac{\mu_{Y}}{a} \leq \frac{g\left(a-\frac{1}{2} h\right)}{a-\frac{1}{2} h}-\frac{g_{0}+\frac{1}{4} d}{a} \leq \frac{-\frac{1}{4} a d+h\left(\frac{1}{2} g_{0}+\frac{1}{8} d\right)}{a\left(a-\frac{1}{2} h\right)}
$$

If we assume that $h$ satisfies the earlier conditions and furthermore $h<\frac{2 a d}{\left(4 g_{0}+d\right)}$, the integrand in (7) starts from negative values, and consequently, the whole integrand is negative and the difference starts from negative values. For the corresponding income distribution, the transformed variable $Y$ does not Lorenz dominate the initial variable $X$. Hence, the continuity of $g(x)$ is also a necessary condition if we demand that the transformed variable should Lorenz dominate the initial variable irrespectively of the distribution $f_{x}(x)$. However, we noted already that the continuity is a necessary condition for the monotone decreasing assumption in 1). From this, it follows that the condition in Theorem 11 ) implies continuity, and hence, the explicit assumption of continuity can be dropped. In a similar way, we can obtain the same result if we study the condition in 2). However, in the case 3) the discontinuity does not jeopardize the monotone increasing property of the quotient $g(x) / x$, and the result in Theorem 13 ) holds even if the function is discontinuous. Therefore, also in this case we can drop the explicit continuity assumption.

Summing up, for arbitrary distributions, $f_{X}(x)$, the conditions in Theorem 11 ), 2), and 3) are both neces- 
sary and sufficient for the dominance relations and the additional assumption about the continuity of the transformation $g(x)$ can be dropped. We obtain a generalized theorem ([1] [2] [6]-[8]).

Theorem 2. Let $X(\geq 0)$ be a random variable with an arbitrary continuous distribution $f_{X}(x)$, mean $\mu_{X}$, and the Lorenz curve $L_{X}(p)$. Let $g(x)$ be a positive, monotone increasing function, let $Y=g(X)$, and let $\mu_{Y}=E(g(X))$ exist. Then the Lorenz curve $L_{Y}(p)$ of $Y$ exists and the following results hold:

1) $L_{Y}(p) \geq L_{X}(p)$ if and only if $g(x) / x$ is monotone decreasing

2) $L_{Y}(p)=L_{X}(p)$ if and only if $g(x) / x$ is constant

3) $L_{Y}(p) \leq L_{X}(p)$ if and only if $g(x) / x$ is monotone increasing.

Remark. From the discussion above, it follows that only in the case 3) can the transformation $g(x)$ be discontinuous.

If we apply these results on income raise policies and on tax policies the transformed variable $Y=g(X)$ is the income after the income raise or after the taxation (cf. e.g. [5] [10]-[12]). We obtain that only income raise policies that (with respect to the initial income) give decreasing relative salary increments result in a decreased income inequality for all initial income distributions. An analogous result holds for progressive tax policies.

\section{Comparison of Two Transformed Variables}

Theorem 1 can be used when the effect of a given tax or salary policy is studied. If several policies are to be compared, the following theorems, which are generalizations of Theorem 1 and Theorem 2, will prove valuable. The generalization of Theorem 1 was first presented by Fellman [10] and proved in [13]. Wilfling [14] later regenerated this theorem. The Hemming-Keen theorem was primarily given in this context. Consider two policies (transformations) $Y=g_{1}(X)$ and $Z=g_{2}(X)$. Following Fellman ([10] [13]), we have

Theorem 3. Let $X$ be a continuous and non-negative random variable with an arbitrary distribution $f_{X}(x)$, mean $\mu_{X}$, and the Lorenz curve $L_{X}(p)$. Let $g_{1}(x)$ and $g_{2}(x)$ be continuous, non-negative and monotone increasing, let $Y=g_{1}(X)$ and $Z=g_{2}(X)$, and let $\mu_{Y}=E\left(g_{1}(X)\right)$ and $\mu_{Z}=E\left(g_{2}(X)\right)$ exist. If the Lorenz curves of $Y$ and $Z$ are $L_{Y}(p)$ and $L_{Z}(p)$, respectively, then the following results hold:

1) $L_{Y}(p) \geq L_{Z}(p)$ if $\frac{g_{1}(x)}{g_{2}(x)}$ is monotone decreasing

2) $L_{Y}(p)=L_{Z}(p)$ if $\frac{g_{1}(x)}{g_{2}(x)}$ is constant

3) $L_{Y}(p) \leq L_{Z}(p)$ if $\frac{g_{1}(x)}{g_{2}(x)}$ is monotone increasing.

Proof: If $\frac{g_{1}(x)}{g_{2}(x)}=C$ (constant), then $Y=C Z$ and the case 2) follows immediately from Theorem 1. If we assume that $\frac{g_{1}(x)}{g_{2}(x)}$ is monotone decreasing for $\mathrm{x}>0$, then $\frac{g_{1}(x)}{g_{2}(x)}-\frac{\mu_{1}}{\mu_{2}}$ attains the value zero only once, being first positive and then negative. Hence, $D(p) \geq 0$ and the case 1) is proved. The case 3 ) can be proved if we let $g_{1}(x)$ and $g_{2}(x)$ exchange their roles and the proof of the case 1) is performed.

Now we study two different salary increase policies.

Example ([13])

1. The salary increases are of the same size regardless of the previous salary

In this case, $y=g_{1}(x)=x+r_{1}$ and the ratio $\frac{g_{1}(x)}{x}=1+\frac{r_{1}}{x}$ is strictly decreasing.

2. The salary increases are of the same size up to a certain salary level, thereafter they are strictly proportional. Now the transformation function is

$$
g_{2}(x)= \begin{cases}x+r_{2} & x<x_{0} \\ x+k_{2} x & x \geq x_{0}\end{cases}
$$


The continuity of $g_{2}(x)$ for $x=x_{0}$ demands that $k_{2} x_{0}=r_{2}$ and $k_{2}=\frac{r_{2}}{x_{0}}$. The ratio $\frac{g_{2}(x)}{x}$ is

$$
g_{2}(x) / x= \begin{cases}1+r_{2} / x & x<x_{0} \\ 1+\frac{r_{2}}{x_{0}} & x \geq x_{0}\end{cases}
$$

and is monotone decreasing.

In both cases, the ratio $g(x) / x$ is monotone decreasing and the policies reduce the income inequality. Now we compare the two policies under the assumption that both give the same increase of the initial mean from $\mu_{0}$ to $\mu_{1}$. For the increased means, we obtain

$$
E\left(g_{1}(X)\right)=\mu_{0}+r_{1}=\mu_{1}
$$

and

$$
\begin{aligned}
\mu_{1} & =E\left(g_{2}(X)\right)=\int_{0}^{x_{0}}\left(x+r_{2}\right) f(x) \mathrm{d} x+\int_{x_{0}}^{\infty}\left(x+r_{2} x\right) f(x) \mathrm{d} x=\int_{0}^{x_{0}}\left(x+r_{2}\right) f(x) \mathrm{d} x+\int_{x_{0}}^{\infty}\left(x+\frac{r_{2}}{x_{0}} x\right) f(x) \mathrm{d} x \\
> & \int_{0}^{x_{0}}\left(x+r_{2}\right) f(x) \mathrm{d} x+\int_{x_{0}}^{\infty}\left(x+\frac{r_{2}}{x} x\right) f(x) \mathrm{d} x=\int_{0}^{x_{0}}\left(x+r_{2}\right) f(x) \mathrm{d} x+\int_{x_{0}}^{\infty}\left(x+r_{2}\right) f(x) \mathrm{d} x=\mu_{0}+r_{2} .
\end{aligned}
$$

If the two increase means should be identical, we obtain the relation

$$
E\left(g_{1}(X)\right)=\mu_{0}+r_{1}=\mu_{1}>\mu_{0}+r_{2} \text { and } r_{1}>r_{2} .
$$

If we apply Theorem 3 on our two policies, we obtain

$$
\frac{g_{1}(x)}{g_{2}(x)}= \begin{cases}\frac{x+r_{1}}{x+r_{2}}=1+\frac{r_{1}-r_{2}}{x+r_{2}} & x<x_{0} \\ \frac{x+r_{1}}{x+\frac{r_{2}}{x_{0}} x}=1+\frac{r_{1}-\frac{r_{2}}{x_{0}} x}{x\left(1+\frac{r_{2}}{x_{0}}\right)} & x \geq x_{0}\end{cases}
$$

Hence, the ratio $\frac{g_{1}(x)}{g_{2}(x)}$ is monotone decreasing for all $x$ and the transformation $g_{1}(x)$ reduces the inequality more than the transformation $g_{2}(x)$.

If we assume that the conditions in Theorem 3 should hold for every income distribution, we can drop the condition that $g_{1}(x)$ and $g_{2}(x)$ are continuous and we can prove in a similar way as above that the conditions are also necessary. We obtain

Theorem 4. Let $X$ be a continuous and non-negative random variable with an arbitrary distribution $f_{X}(x)$, mean $\mu_{X}$, and the Lorenz curve $L_{X}(p)$. Let $g_{1}(x)$ and $g_{2}(x)$ be non-negative and monotone increasing, let $Y=g_{1}(X)$ and $Z=g_{2}(X)$, and let $\mu_{Y}=E\left(g_{1}(X)\right)$ and $\mu_{Z}=E\left(g_{2}(X)\right)$ exist. If the Lorenz curves of $Y$ and $Z$ are $L_{Y}(p)$ and $L_{Z}(p)$, respectively, then the following results hold:

1) $L_{Y}(p) \geq L_{Z}(p)$ if and only if $\frac{g_{1}(x)}{g_{2}(x)}$ is monotone decreasing

2) $L_{Y}(p)=L_{Z}(p)$ if and only if $\frac{g_{1}(x)}{g_{2}(x)}$ is constant

3) $L_{Y}(p) \leq L_{Z}(p)$ if and only if $\frac{g_{1}(x)}{g_{2}(x)}$ is monotone increasing.

In a similar way as above, we obtain that the discontinuities in $g_{1}(x)$ and $g_{2}(x)$ can only be finite posi- 
tive jumps. If the condition in Theorem 4 1) holds, then $g_{2}(x)$ can be discontinuous, but $g_{1}(x)$ can be discontinuous only at such points where $g_{2}(x)$ is discontinuous, and additionally, the corresponding jumps must be such that $\frac{g_{1}(x)}{g_{2}(x)}$ is monotone decreasing. In 2) $g_{2}(x)$ and $g_{1}(x)$ can be discontinuous only at the same points, and additionally, the corresponding jumps must be such that $\frac{g_{1}(x)}{g_{2}(x)}$ remains constant. In 3) $g_{1}(x)$ can be discontinuous, but $g_{2}(x)$ can be discontinuous only at such points where $g_{1}(x)$ is discontinuous, and additionally, the corresponding jumps must be such that $\frac{g_{1}(x)}{g_{2}(x)}$ is monotone increasing.

Remark. Theorems 3 and 4 are generalized versions of Theorems 1 and 2, respectively. This is clear if we introduce the simplified condition $g_{2}(x)=x$.

\section{Conclusions}

Redistributions of income have commonly been defined as transformations of the initial income variable. The transformations are mainly considered as tax or transfer policies yielding post-tax or post-transfer incomes, and therefore, the transformations are usually assumed to be positive, monotone increasing, and continuous. Recently, discontinuous transformations have been discussed. Particularly, we were interested in determining if one can drop the assumptions of continuity of the transformations.

In this study, we considered the effect of variable transformations on the redistribution of income. The aim was to compare and generalize the conditions considered in earlier papers. The fundamental concern has been the Lorenz ordering between the initial and transformed income. We have obtained that, if we demand sufficient and necessary conditions, theorems earlier obtained still hold and the continuity assumption can be implicitly included in the general conditions. Especially, we have considered the optimal cases that the transformed variable Lorenz dominates the initial one. In applications, this case is important because it yields policies which reduce income inequality. The main result is that continuity is a necessary condition if income inequality should remain or be reduced.

Empirical applications of the optimal policies of classes of transfer policies and of tax policies considered here have been discussed in Fellman et al. [12] [15]. There we developed "optimal yardsticks" to gauge the effectiveness of given real tax and transfer policies in reducing inequality.

\section{Acknowledgements}

This study was supported by grants from the Finnish Society of Sciences and Letters and Magnus Ehrnrooth Foundation.

\section{References}

[1] Jakobsson, U. (1976) On the Measurement of the Degree of Progression. Journal of Public Economics, 5, 161-168. http://dx.doi.org/10.1016/0047-2727(76)90066-9

[2] Fellman, J. (2009) Discontinuous Transformations, Lorenz Curves and Transfer Policies. Social Choice and Welfare, 33, 35-342. http://dx.doi.org/10.1007/s00355-008-0362-4

[3] Hemming, R. and Keen, M.J. (1983) Single Crossing Conditions in Comparisons of Tax Progressivity. Journal of Public Economics, 20, 373-380. http://dx.doi.org/10.1016/0047-2727(83)90032-4

[4] Fellman, J. (2011) Discontinuous Transfer Policies with Given Lorenz Curve. Advances and Applications in Statistics, 20, 133-141.

[5] Fellman, J. (1976) The Effect of Transformations on Lorenz Curves. Econometrica, 44, 823-824. http://dx.doi.org/10.2307/1913450

[6] Kakwani, N.C. (1977) Applications of Lorenz Curves in Economic Analysis. Econometrica, 45, 719-727. http://dx.doi.org/10.2307/1911684

[7] Fellman, J. (2013) Properties of Non-Differentiable Tax Policies. Theoretical Economics Letters, 3, 142-145. http://www.scirp.org/journal/tel 
http://dx.doi.org/10.4236/tel.2013.33022

[8] Fellman, J. (2013) Discontinuous Transformations and Lorenz Curves. Journal of Statistical and Econometric Methods, 2, 151-160.

[9] Lambert, P.J. (1993) The Distribution and Redistribution of Income. 3rd Edition, University Press, Manchester, 19, 313 p.

[10] Fellman, J. (1977) The Effect of Transformations on Lorenz Curves. Zbl Math, 336, 408.

[11] Fellman, J. (1995) Intrinsic Mathematical Properties of Classes of Income Redistributive Policies. Swedish School of Economics and Business Administration Working Paper 306, 26 p.

[12] Fellman, J., Jäntti, M. and Lambert, P.J. (1996) Optimal Tax-Transfer Systems and Redistributive Policy: The Finnish Experiment. Swedish School of Economics and Business Administration Working Paper 324, 16 p.

[13] Fellman, J. (1980) Transformations and Lorenz Curve. Swedish School of Economics and Business Administration Working Paper 48, 18 p.

[14] Wilfling, B. (1996) Lorenz Ordering of Generalized Beta-II Distributions. Journal of Econometrics, 71, 381-388. http://dx.doi.org/10.1016/0304-4076(94)01710-7

[15] Fellman, J., Jäntti, M. and Lambert, P.J. (1999) Optimal Tax-Transfer Systems and Redistributive Policy. The Scandinavian Journal of Economics, 101, 115-126. http://dx.doi.org/10.1111/1467-9442.00144 